\title{
Coronavirus Disease 2019 (COVID-19) in General Medicine: A Clinical-Epidemiological Case Series Reporting of 16 Patients in Toledo (Spain), March-May 2020 also in Covid-19, "If you Hear Hoofbeats, Think Horses not Zebras"
}

\section{Turabian JL*}

Specialist in Family and Community Medicine, Regional Health Service of Castilla la Mancha (SESCAM), Spain

*Corresponding author: Jose Luis Turabian, Health Center Santa Maria de Benquerencia. Regional Health Service of Castilla la Mancha (SESCAM), Toledo, Spain, Email: jturabianf@

\section{Research Article}

Volume 4 Special Issue 1

Received Date: July 02, 2020

Published Date: July 31, 2020

DOI: $10.23880 /$ eij-16000S1-006 hotmail.com

\section{Abstract}

Objective: Describe clinical-epidemiological characteristics that can help the general practitioner to consider the diagnosis of COVID-19

Methodology: A retrospective and descriptive study was carried out on clinical and epidemiological variables in patients diagnosed with COVID-19 who consulted in general medicine office at a health center in Toledo, Spain, from March 1 to May 31, 2020.

Results: 17 patients were included, with a mean age of 48 years, 7 men and 10 women, 2 were asymptomatic throughout the period and their diagnosis was made after a contact. The most frequent symptoms were: fever $67 \%$, cough $67 \%$, dyspnea $45 \%$, myalgia $40 \%$, and headache $40 \% .18 \%$ presented pneumonia. The mean duration of symptoms was 25 days. The severity was $76 \%$ mild, and $12 \%$ critical. There were 2 deaths. $82 \%$ reported prior known contact. The secondary attack rate was $12 \%$. The number of chronic diseases was 25 in the 17 patients, being the most frequent groups, endocrine with 52\%, and circulatory system with $16 \% .47 \%$ were workers in socio-health centers.

Conclusion: In the context of general medicine in Toledo (Spain), during the exponential growth phase and subsequent decrease in cases, of the COVID-19 outbreak (March-May 2020), the symptoms previously described as common were the most frequent (fever, cough, and dyspnea) and the obvious epidemiological risk factors (such as being a social or health worker and having been in contact with a case) were frequent. The first thing that should be taken into account, which seems obvious, in general medicine and epidemiology, also for COVID-19, is that the most frequent symptoms and risk factors must be considered first.

Keywords: Coronavirus; COVID-19; SARS-CoV-2; General Practice; Epidemiology; Disease \& Medicine; Symptoms; Severity

Abbreviations: COVID-19: Coronavirus Disease 2019; SARS-Cov-2: SARS-Coronavirus-2; IC: Intensive Care; GP: General Practitioners; PCR: Polymerase Chain Reaction; ACE: Angiotensin-Converting-Enzyme; Arbs: Angiotensin-
Receptor Blockers; NICE: National Institute for Health and Care Excellence; CDC: Centers for Disease Control and Prevention; ARI: Acute Respiratory Infections. 


\section{Epidemiology International Journal}

\section{Introduction}

In December 2019, a cluster of pneumonia cases emerged in Wuhan City, Hubei Province, China. The coronavirus disease 2019 (COVID-19) is caused by the SARScoronavirus-2 (SARS-CoV-2), a virus primarily zoonotic. WHO declared COVID-19 a global pandemic and a public health emergency. The spread of COVID-19 epidemic is unprecedented and it continues to spread affecting many countries and territories around the world [1-4]. Its numbers are changing rapidly, and as of this writing, as of June 29 , 2020 , the pandemic has infected more than $10,000,000$ people and killed more than 500,000 worldwide; The United States has more than 2,500,000 cases, followed by Brazil with more than $1,000,000$, Russia with more than 640,000 , India with more than 548,000, United Kingdom with more than 313,000 , Peru with more than 279,000 , Chile with more than 271,000. Spain with more than 248,000 , Italy with more than 240,000, and Iran with more than 225,000 [5].

Presentations of COVID-19 have ranged from asymptomatic/mild symptoms to severe illness and mortality. Common symptoms have included fever, cough, and shortness of breath. Other symptoms, such as malaise and respiratory distress, have also been described [6-8]. As the COVID-19 pandemic evolves, we gradually are accumulating knowledge to guide patient care. Although most disease is mild $(80-85 \%), 12-15 \%$ have a serious illness, and between $1-5 \%$ a potentially fatal disease, which can require intensive care (IC) for issues such as acute respiratory distress syndrome, shock, and multiorgan failure $[9,10]$. Results from various specialist groups around the world are beginning to converge on the true case fatality rate of COVID-19, which appears to be around 1 percent [11].

Recognizing that lack of preparation for an outbreak is a major global health challenge, sharing knowledge on how to care for the minority of patients who become critically ill is important. In Italy, previously "normal" hospitals have been transformed into giant intensive care units. But it is also very important how to deal with infection in primary care and in the community, which means attending to most cases [12].

Spain, with more than 11,000 positives and 500 deaths (as of March 18, 2020) [13] was the second country in the world that registers more new cases every day. The virus was in Spain in exponential growth phase and showed a similar rate to that of Italy. At the time, the healthcare system was unable to cope with the massive influx of infectious cases to emergency departments and hospitals. In this way, patients with mild symptoms should stay at home and in their community whenever possible. This enormous task fell to the general practitioners (GP), other health and non-health personnel from Primary Care and the local community itself.
For most illnesses the GP is the first point of contact in the health care system and he looks after a population whose age and sex composition is known. The essence of general medicine is to assist individuals in families and communities, and this implies, on the one hand a good continuity of care, and moreover a knowledge of the nature of diseases in the community $[14,15]$. The GP is in a rare position that combines the individual and community dimensions, and there is a great need to extend the clinical horizons to the epidemiological and community aspects of care. The "path of all patients" begins and ends with the GP [16,17]. All these characteristics of the GP can also be applied and are affected by the current outbreak of COVID-19.

In this scenario, although there is a growing number of publications presenting the data of COVID-19 patients, many clinical and epidemiological characteristics remain unknown, and most of the data comes from the most seriously ill patients who are admitted to hospitals and even to intensive care $[18,19]$, but there is a lack of descriptions of COVID-19 patients at the general medicine level, where they are cared for by GPs [20]. That is, primary care research on the characteristics and outcomes of suspected COVID-19 cases may provide a different view of the COVID-19 epidemic [21].

This study compiles 17 successive cases of COVID-19 attended in the general medicine consultation of a health center in Toledo (Spain) from March 1 to May 31, 2020 (from the start of the exponential outbreak until the number of new infected decreased), with the aim of exposing and summarizing their clinical and epidemiological data.

\section{Materials and Methods}

A retrospective and descriptive study of patients diagnosed with COVID-19 who were treated at a general medicine consultation in the Santa Maria de Benquerencia Health Center, Toledo, Spain, was conducted, which has a list of 2,000 patients> 14 years of age (in Spain, GPs care for people $>=14$ years of age), from March 1, 2020 to May 31, 2020 (date the case growth curve began to flatten). March 1 was chosen as the beginning of the study, since on that date 6 official cases of COVID-19 were reported in the CastillaLa Mancha region, which has a population of 2,035,505 inhabitants, whose capital is Toledo with 687,084 inhabitants (2018), and it is from that day when official statistics indicate an exponential increase in new cases. In the province of Toledo (Spain) where the study was conducted, on March 10, 2020, 6 cases were counted, on March 16, 133, on April 1, 1593, on April 15, 3193, on May 1, 4744, and May 30, 5499.

The diagnosis of COVID-19 was made by polymerase chain reaction (PCR) (oropharyngeal) or IgG and IGM 


\section{Epidemiology International Journal}

serology for SARS-COV-2 (IgM positive and IgG positive or IgM negative and IgG positive). Newly diagnosed patients were followed until their cure with negative PCR.

Because of, since the outbreak began, diagnostic tests were not available at the primary care level, since the instructions were as of March 18, 2020, that diagnostic test for SARS-CoV-2 be performed at people with a clinical picture of acute respiratory infection admitted to the hospital, or respiratory infection of any degree in health personnel. Similarly, routine diagnostic tests are not performed on contacts $[15,19]$. And, since May 19, 2020 PCR began to be carried out on suspected COVID-19 cases in general medicine. So, there were patients who were diagnosed in the hospital and others who were diagnosed at the primary care level.

Only cases of patients who belonged to the office list of patients and for whom data were available on their entire clinic course, from start to cure (hospital data including), were included; During this period, other cases of COVID-19, belonging to other consultations, were attended occasionally due to the absence of their usual GP, but these patients, for whom data were not available for the entire course of their evolution, were not included in the study.

The following variables were collected COVID-19 diagnostic test, age, sex, socio-health worker, symptoms, severity, hospitalization, previous contacts, secondary contagion to another person, previous treatment with angiotensin-converting-enzyme (ACE) inhibitors or angiotensin-receptor blockers (ARBs), sick leave, days duration of symptoms, and chronic diseases (defined as "any alteration or deviation from normal that has one or more of the following characteristics: is permanent, leaves residual impairment, is caused by a non-reversible pathological alteration, requires special training of the patient for rehabilitation, and / or can be expected to require a long period of control, observation or treatment" [22-24], classified according to the International Statistical Classification of Diseases and Health-Related Problems, CD10 Version: 2019 [25].

The severity classification was based on 4 levels according to severity of symptoms: mild, moderate, severe and critical. Mild patient that only showed as mild symptoms in without radiographic feature; Moderate patient showed as fever, respiratory symptoms \& radiographic feature; Severe cases of meet one of three criteria such as dyspnea, respiratory rate $>30$ times/min; oxygen saturation $<93 \%$ in ambient air; PaO2/FiO2 <300 mmHg; For critical patient also meet one of three criteria such as respiratory failure; septic shock; multiple organ failure [26]. The classification of these cases to mild, moderate and severe depends on a subjective evaluation that in some cases was carried out remotely [21].
In the cases that required hospitalization, hospital reports were used to obtain the data. Following previous communications on the minor importance of the radiological examination as a diagnostic tool [27,28], X-rays and other imaging tests were not performed systematically in all cases.

Guidelines for the management of suspected or confirmed cases in the community were followed. These guidelines recommend minimizing personal consultation and limited examination unless necessary (limited examination means that auscultation is not essential, and a rapid diagnosis of pneumonia could be made based on confusion, temperature, respiratory rate and heart rate, which are the clinical observations that are readily available to professionals in primary health care). So, the most of the consultations with patients were remote or telemedicine consultations $[29,30]$.

\section{Results}

Seventeen patients were included; 11 of them were diagnosed with PCR, and 6 with IgM and IgG. The mean age was 48 years (range: $27-66$ years), 7 were men and 10 women, 2 were asymptomatic throughout the period and their diagnosis was made after contact with a sick person.

\begin{tabular}{|c|c|}
\hline Symptoms & Number (Total = 15 patients) \\
\hline Fever & 10 \\
\hline Cough & 10 \\
\hline Dyspnea & 7 \\
\hline Myalgia & 6 \\
\hline Headache & 6 \\
\hline Asthenia & 4 \\
\hline Nausea / Vomiting & 4 \\
\hline Anxiety & 4 \\
\hline Insomnia & 4 \\
\hline General discomfort & 4 \\
\hline Anosmia / Ageusia & 4 \\
\hline Diarrhea & 3 \\
\hline Odynophagia & 3 \\
\hline Chest pain & 2 \\
\hline Anorexia & 1 \\
\hline Dysphagia & 1 \\
\hline Rhinorrhea & 1 \\
\hline Chilblains / Flictenas & 1 \\
\hline Petechiae & 1 \\
\hline Upper limb tremor & 1 \\
\hline
\end{tabular}

Table 1: Chronic Diseases (Who, Icd-10 Groups) In the Case Series. 
The symptoms in the 15 patients with symptoms were (Table 1, Figure 1): 67\% fever, 67\% cough, 45\% dyspnea, $40 \%$ myalgia, $40 \%$ headache, $27 \%$ asthenia, $27 \%$ nausea / vomiting, $27 \%$ anxiety , $27 \%$ insomnia, $27 \%$ general malaise, $27 \%$ anosmia / ageusia, 20\% diarrhea, 20\% odynophagia, $13 \%$ chest pain, $7 \%$ anorexia, 7\% dysphagia, $7 \%$ rhinorrhea, $7 \%$ chilblains / gallbladders, 7\% petechiae, 7\% upper limb tremor. Radiologically verified pneumonia was found in $18 \%$ of the 17 cases ( $20 \%$ in cases with symptoms), mean duration of symptoms 25 days (range: 10-54 days). There were 2 deaths (both males 61 and 62 years, respectively) and occurred in patients with pneumonia and admitted to the hospital.

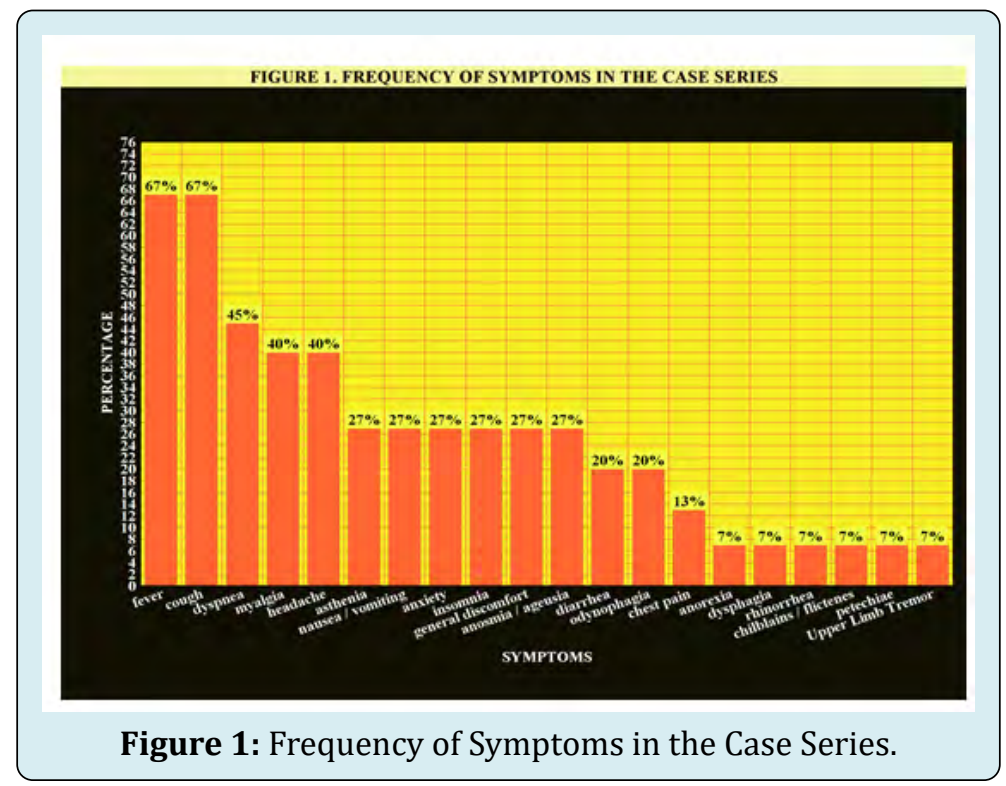

Of the 17 cases, 5 were hospitalized. The severity of the 17 cases was classified as: 76\% Mild, 12\% critical, 6\% Moderate, and 6\% Severe. They reported $82 \%$ known prior contact, and secondary infection in another person they had contact with in $12 \% .18 \%$ were on previous treatment with ACE inhibitors or ARBs. The number of chronic diseases was 25 in the 17 patients included; the most frequent groups were endocrine with $52 \%$, and the circulatory system with $16 \%$
(Table 2, Figure 2). As complications or events appearing in the course of the disease, one patient presented truncated mourning (death of her husband by COVID-19), another patient presented renal colic, another gout attack, and another more pyelonephritis. Of the 17 patients included, $47 \%$ were health workers or worked in socio-health centers. 12 patients $(70 \%)$ required sick leave.

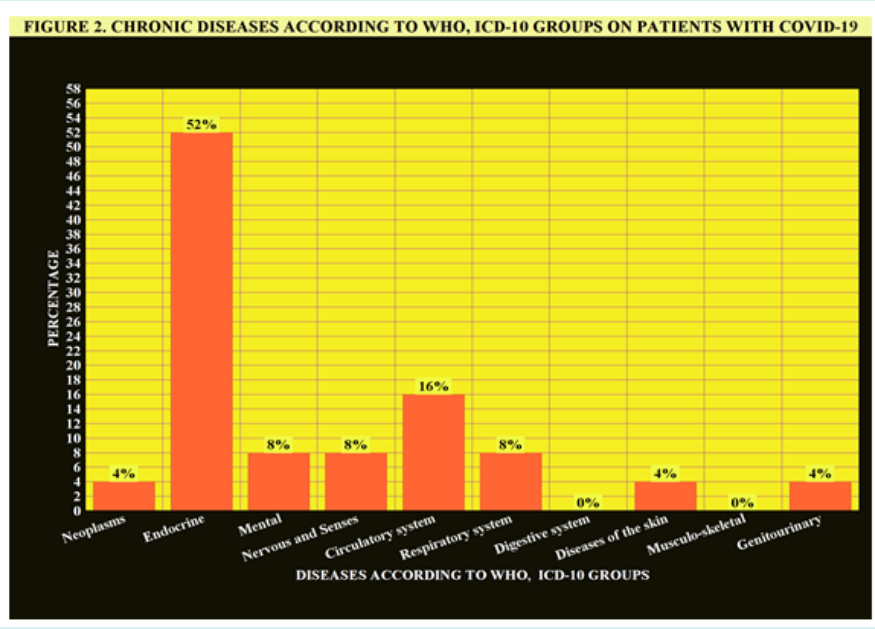

Figure 2: Percentage of Chronic Diseases (WHO, Icd-10 Groups) in the Case Series. 


\section{Epidemiology International Journal}

\begin{tabular}{|l|c|}
\hline $\begin{array}{c}\text { Diseases According to WHO, } \\
\text { ICD-10 Groups }\end{array}$ & $\begin{array}{c}\text { Number of chronic } \\
\text { diseases in patients } \\
\text { with COVID-19 N=25 } \\
\text { chronic diseases ( ) } \\
\text { indicates \% }\end{array}$ \\
\hline -II Neoplasms & $1(4)$ \\
\hline -IV Endocrine & $13(52)$ \\
\hline -V Mental & $2(8)$ \\
\hline -VI-VIII Nervous and Senses & $2(8)$ \\
\hline -IX Circulatory system & $4(16)$ \\
\hline -X Respiratory system & $2(8)$ \\
\hline -XI Digestive system & 0 \\
\hline -XII Diseases of the skin & $1(4)$ \\
\hline -XIII Musculo-skeletal & 0 \\
\hline -XIV Genitourinary & $1(4)$ \\
\hline
\end{tabular}

Table 2: Number of Chronic Diseases According to WHO, Icd10 Groups on Patients with Covid-19.

\section{Discussion}

\section{Age, Sex / Gender, Occupation, Previous Contact and Secondary Contagion}

Our series of patients is relatively young, as is the demography of the area served in the consultation under study. Women predominated (59\%), which may be related to the high percentage of social and health workers in our series (44\% health workers or work in socio-health centers). This data is in line with the statement for Spain, where as of May 29,2020 , it has been reported that 51,482 health workers have been infected since the outbreak began [31]; which represents, with 238,564 patients infected on that date, $22 \%$ of the cases (almost 1 in 4). In this sense, in Spain, being a social or health worker is a fact that directs the diagnosis of respiratory symptoms during the epidemic period clearly towards COVID-19 [32].

One of the most overlooked aspects of gender in illness, including COVID-19, is the near-universal role of women in the center of families and networks, compounded by the fact that most health workers are women [33]. According to WHO data, of the more than 8,000 SARS cases - 2003 epidemic - more than half of the infected people were women, and $21 \%$ of the cases affected precisely those who worked in the health field $[34,35]$. These high frequency occurrences of cases in women and social or health workers explain the high number of previous contact known with COVID-19: these were personnel from health centers and nursing homes, who at the start of the outbreak acted as vectors of the disease
[36-41].

We found a known secondary attack rate of $12 \%$. The figures reported so far are between $0.45 \%$ between close contacts, $3 \%-10 \%-25 \%$ for cohabitants of the same family, and up to $35 \%$ of secondary infections as a consequence of short-term social events [37].

\section{Clinical presentation}

Common signs in presentation include fever, cough, and shortness of breath. Although the disease can be asymptomatic or mild in many cases, moderate cases require hospitalization and severe cases require the support of vital organ functions. WHO advised suspecting COVID-19 in patients with acute fever and $\geq 1$ sign or symptom of respiratory disease combined with an exposure risk factor [21]. According to the National Institute for Health and Care Excellence (NICE), the typical symptoms are cough, fever, and loss of sense of smell or taste, but they may also have breathlessness (which may cause anxiety), delirium (which may cause agitation), fatigue, headache, muscle aches and sore throat [30].

Furthermore, a summary of the clinicopathological characteristics of patients affected by COVID-19 has been published. According to these data, a higher proportion of infected patients were male (53\%), and the most commonly experienced symptoms were fever and cough. Myalgia, anorexia, chest tightness and dyspnoea were found in some patients. A relatively small percentage of patients were asymptomatic. According to a meta-analysis, the two major symptoms observed were fever (78.4\%) and cough (58.3\%). Other common symptoms included fatigue (34\%), myalgia (21.9\%), expectoration $(23.7 \%)$, anorexia $(22.9 \%)$, chest tightness $(22.9 \%)$ and dyspnoea (20.6\%). Minor symptoms included nausea and vomiting (6.6\%), diarrhoea (8.2\%), headache (11.3\%), pharyngalgia (11.6\%), shivering (15.2\%) and rhinorrhea (7.3\%). About $5.4 \%$ of the patients were asymptomatic [42]. Other systematic reviews of studies of patients with COVID-19 indicated also a high incidence of fever $(>88 \%)$ and cough $(>68 \%)$ [43,44]. Our results coincide in the most frequent symptoms.

The Centers for Disease Control and Prevention (CDC) has long said that fever, cough and shortness of breath are indications that someone might have the disease caused by the novel coronavirus. In late April 2020 were added six more conditions that may come with the disease: chills, repeated shaking with chills, muscle pain, headache, sore throat and loss of taste or smell [45]. So, scientists document a growing list of atypical coronavirus infection symptoms, giving doctors more insight into the emerging disease. However, this list of typical and infrequent symptoms can 


\section{Epidemiology International Journal}

lead to diagnostic difficulties.

In this sense, an increasing series of rare or atypical symptoms have been described: more than two thirds of patients (64-87\%) with mild COVID-19 reported alterations in their sense of smell or taste [46-48]; a multi-system inflammatory syndrome (symptoms similar to toxic shock syndrome or Kawasaki disease), strokes (studies of The Netherlands and France suggest that clots appear in 20\% to $30 \%$ of patients with critically ill COVID-19) [49-51], deep vein thrombosis (in $65 \%$ of 34 patients with COVID-19 who underwent venous ultrasound upon admission to an intensive care unit) [52], neurological symptoms (36\%) (Headache, dizziness, languidness, unstable walking, and malaise, cerebral haemorrhage, cerebral infarction, and other neurological diseases) [53], rashes or purpura, lesions resembling frostbite or chilblains, and other dermatological symptoms [54,55], silent hypoxia (extremely low blood oxygen levels without accompanying dyspnoea) [56], symptom gastrointestinal $(32 \%$ of patients have been reported to experience mild gastrointestinal symptoms including loss of appetite, abdominal pain 4\%, diarrhoea $8 \%$ and nausea / vomiting: $8 \%$ and diarrhoea) [57,58], cardiovascular symptoms (chest pain and ST elevation; cardiogenic shock and acute respiratory distress with deep hypoxia; or de-compensation of previous heart disease) [59], and psychiatric symptoms (most studies evaluated SARSCoV and MERS-CoV infections in hospitalized patients) such as delirium, depression, anxiety, fatigue, and post-traumatic stress disorder [60-62]. However, some of these atypical symptoms were found in our small series, but they were rare, or were not present.

COVID-19 was initially understood as pneumonia caused by SARS-CoV-2. Now, after having reported a large number of mild and asymptomatic cases of COVID-19 (the so-called iceberg phenomenon that is common for most infectious diseases) and the institution of PCR as a diagnostic standard, the radiological examination seems less important as a diagnostic tool than it used to be; Radiological examinations may not be necessary when caring for large numbers of patients, when radiological examinations are not available, or when patients may be diagnosed with mild COVID-19 by an experienced physician (although patients with COVID- 19 often have lung lesions detectable by tomography) [27,28]. In this sense, in Spain up to 54\% of cases diagnosed until May 10 developed pneumonia [63], although this percentage does not take into account undiagnosed patients (without PCR; milder or asymptomatic cases). In our case series, including mild and asymptomatic cases, $18 \%$ showed radiological verified pneumonia.

In the same consultation where this study was carried out, previously the clinic of patients with COVID-19 was compared with that of patients with acute respiratory infections (ARI), during the COVID-19 outbreak, in the period when the PCR test for the diagnosis in general medicine was not available: the suggesting symptoms of COVID-19 in ARIs, coincide with the current results: fever, dyspnoea, presence of chronic diseases of the circulatory system, selfcommunication of possible contact with a COVID-19 patient, or being a health professional / working in healthcare settings [32].

\section{Asymptomatics, Severity, Chronic Diseases}

In our series, $12 \%$ were asymptomatic. This is a lower figure than that shown by the seroprevalence study in Spain (26\% were asymptomatic people) [64], and that reported by other studies in which the prevalence of asymptomatic disease is estimated between 40 and $45 \%$ of people who test positive for SARS-CoV-2 [65]. This difference is logical since our cases are patients who come to the general medicine consultation, not a population sample. It has been reported that most patients with suspicious diagnosis and isolation at home are presumed to have good results [21]. In our study, it seems to be confirmed: of the 17 cases, $76 \%$ were Mild; but we must not forget that $12 \%$ were critical, $6 \%$ Moderate, and $6 \%$ Severe, and there were 2 exitus.

Our study found that the most frequent groups of chronic diseases were endocrine with $52 \%$ and circulatory system with $16 \%$. It has been reported that diabetes occurred in $42 \%$ of patients with COVID-19 [66], and persons with diabetes had a significantly greater risk for severe pneumonia, release of tissue injury-related enzymes, excessive uncontrolled inflammatory responses, and hypercoagulable state associated with deregulated glucose metabolism compared with persons with no diabetes [67]. Further, approximately 1 in 10 patients with COVID-19 and diabetes has been reported to die within 7 days of hospital admission [68]. On the other hand, hypertension was associated with increased poor outcome, including mortality, in patients with COVID-19 [69].

There is controversy over whether treatment with ACE inhibitors or ARBs in COVID-19 is a risk factor or by cons has clinical utility. On theoretical grounds, blockade of ACE-2 which primarily metabolises angiotensin II into angiotensin-(1-9), could confer anti-infective properties against SARS-CoV-2 by preventing entry of the virus into lung pneumocytes [70]. In our study, $18 \%$ of patients were on treatment with ACEI / ARA II.

\section{Study Limitations}

- Obviously it is a small study (in any case it is a descriptive study, not an analytical one and tests of statistical significance are not used), and thus may not 


\section{Epidemiology International Journal}

have collected symptoms that appear infrequently. In other words, our study may not be useful to study rare symptoms (the probability of finding a rare case is small). However, that very fact of the small size of the study may make the results reliable for frequent symptoms that appear in the vast majority of patients.

- Our data are not fully representative of the general population. As, with other studies in primary care, such as the report on the characteristics of the first 3802 people evaluated for SARS-CoV-2 within the primary care surveillance network. sentinel of the Royal College of General Practitioners [71], those cases with very mild symptoms or no symptoms that they do not consult are excluded and, instead, they reflect consultation patterns where there are an excessive representation of women and, in our case, young people (reproducing the demographics of the neighborhood served by the study consultation).

\section{Conclusion}

In the context of general medicine in Toledo (Spain), during the exponential growth phase and subsequent decrease in cases, of the COVID-19 outbreak (March-May 2020), the symptoms previously described as common or usual were the most frequent (fever, cough, and dyspnea), and the severity was mostly mild, but some rarer symptoms (such as anosmia/ageusia, diarrhea, odynophagia, chest pain, anorexia, dysphagia, rhinorrhea, chilblains / gallbladders, and petechiae) also they appeared infrequently, and a few portions of patients developed severe pneumonia with progression to lethal complications. Obvious epidemiological risk factors (such as being a social or health worker and having been in contact with a case) were frequent.

When a patientcomes to the consultation with a complaint without diagnostic affiliation, one of the key challenges that the general practitioner often faces is deciding the extent and depth of the diagnostic study to be carried out, the diagnoses to be considered and the degree of uncertainty assume. An example of this situation is reflected in COVID-19. In order to address this type of clinical-epidemiological dilemma, the first consideration that must be taken into account, which seems more than obvious, is that common symptoms and usual epidemiological risk factors are frequent and the rare are infrequent. This does not justify the fact that the latter are systematically forgotten in the epidemiological-clinical study, but of course it does not mean always including them in the differential diagnosis. In COVID-19, too, we must first think about the most frequent problems, with a nuance of prudence. This recommendation has been reflected in the popular aphorism: "If you hear hoofbeats, think horses not zebras" [72], but we are also obliged to think about those exceptional, but serious diseases [73].

\section{References}

1. Gupta SD (2020) Coronavirus Pandemic: A Serious Threat to Humanity. J Health Manag 22(1): 1-2.

2. Zhu N, Zhang D, Wang W, Li X, Song J, et al. (2020) A novel coronavirus from patients with pneumonia in China, 2019. N Engl J Med 382(8): 727-733.

3. Garcia Basteiro AL, Chaccour C, Guinovart C, Llupia A, Brew J, et al. (2020) Monitoring the COVID-19 epidemic in the context of widespread local transmission. Lancet Respir Med 8: 440-442.

4. Mackenzie JS, Smith DW (2020) COVID-19-A Novel Zoonotic Disease: A Review of the Disease, the Virus, and Public Health Measures. Asia Pac J Public Health.

5. COVID-19 Dashboard by the Center for Systems Science and Engineering (CSSE) at Johns Hopkins University.

6. Cennimo DJ (2020) Coronavirus Disease 2019 (COVID-19) Clinical Presentation. Medscape.

7. Gandhi RT, Lynch JB, Del Rio C (2020) Mild or Moderate Covid-19. N Engl J Med.

8. Berlin DA, Gulick RM, Martinez FJ (2020) Severe Covid-19. N Engl J Med.

9. Wu Z, McGoogan JM (2020) Characteristics of and important lessons from the coronavirus disease 2019 (COVID-19) outbreak in China: summary of a report of 72314 cases from the Chinese Center for Disease Control and Prevention. JAMA 323(13): 1239-1242.

10. Baud D, Qi X, Nielsen Saines K, Musso D, Pomar L, et al. (2020) Real estimates of mortality following COVID-19 infection. Lancet Infect Dis 20: 773.

11. Murthy S, Gomersall CD, Fowler RA (2020) Care for critically ill patients with COVID-19. JAMA 323(15): 1499-1500.

12. Smith J, Sears N, Taylor B, Johnson M (2020) Serious games for serious crises: reflections from an infectious disease outbreak matrix game. Globalization and Health 16: 18.

13. (2020) Coronavirus (COVID-19). Seguridad Nacinal.

14. Turabian JL (2017) The Variation of Seasonal Diseases in Family Medicine Depends on Infectious Diseases and these are Mainly Respiratory Diseases. J Gen Pract 5(3): 1000309.

15. Turabian JL (2020) Micro-Impact of the Pandemic 


\section{Epidemiology International Journal}

by Covid-19 in the General Medicine: Clinical and Epidemiological Reflections from the Situation in Spain March 2020. Epidemol Int J 4(2): 000141.

16. Turabian JL (2017) Prevalence of Chronic Diseases: in Defence of Epidemiological Craftsmanship in Family Medicine. CP Epidemiology 1(1): 005.

17. Turabian JL (1995) Family and community medicine notebooks.

18. Argenziano MG, Bruce SL, Slater CL, Tiao JR, Baldwin MR, et al. (2020) Characterization and clinical course of 1000 patients with coronavirus disease 2019 in New York: retrospective case series. BMJ 369: 1996.

19. Petrilli CM, Jones SA, Yang J, Rajagopalan H, O’Donnell L, et al. (2020) Factors associated with hospital admission and critical illness among 5279 people with coronavirus disease 2019 in New York City: prospective cohort study. BMJ 369: 1966.

20. De Lusignan S, Dorward J, Correa A, Jones N, Akinyemi O, et al. (2020) Risk factors for SARS-CoV-2 among patients in the Oxford Royal College of General Practitioners Research and Surveillance Centre primary care network: a cross-sectional study. Lancet Infect Dis, pp: 1-9.

21. Hamed E, Abd Elhamid M, Alemrayat B (2020) Suspected cases of COVID-19: study protocol for reporting characteristics and the outcomes. Family Medicine and Community Health 8(2): 000400.

22. Strauss AL (1984) Chronic illness and the quality of life.

23. Gill M (2017) Augmented care: An alternative model of care for people with chronic disease. Int J Care Coord 20(1-2): 5-7.

24. Calderon Larranaga A, Vetrano DL, Onder G, Gimeno Feliu LA, Coscollar Santaliestra C, et al. (2016) Assessing and Measuring Chronic Multimorbidity in the Older Population: A Proposal for Its Operationalization. J Gerontol A Biol Sci Med 72(10): 1417-1423.

25. (2019) International Statistical Classification of Diseases and Health-Related Problems $10^{\text {th }}$ Version.

26. (2020) Report of the WHO-China Joint Mission on Coronavirus Disease 2019 (COVID-19), pp: 1-40.

27. Qiu H, Wu J, Hong L, Luo Y, Song Q, et al. (2020) Clinical and epidemiological features of 36 children with coronavirus disease 2019 (COVID-19) in Zhejiang, China: an observational cohort study. Lancet Inf Dis 20: 689-696.
28. Chen D, Tang F, Lu S, Song Q (2020) Toward a clinically based classification of disease severity for paediatric COVID-19. Lancet Inf Dis, pp: 1.

29. (2020) COVID-19 rapid guideline: managing suspected or confirmed pneumonia in adults in the community. National Institute for Health and Care Excellence (NICE).

30. (2020) COVID-19 rapid guideline: managing symptoms (including at the end of life) in the community. National Institute for Health and Care Excellence (NICE).

31. Millan A (2020) Health confirms that 51,482 health workers have been infected since the start of the crisis. Diario16.

32. Turabian JL (2020) Clinical-Epidemiological Characteristics That May Help the General Practitioner to Consider Covid-19 Diagnosis in Acute Respiratory Infections When Diagnostic Tests Are Not Accessible. Epidemol Int J 4(2): 000143.

33. Chada AS (2020) Are men more affected by the novel coronavirus than women: Insights into why more men than women are affected by the novel coronavirus. Roche.

34. (2020) SARS (Severe Acute Respiratory Syndrome). World Health Organization (WHO).

35. Turabian JL (2020) Sex and Gender Bio-psychosocial Differences in Coronavirus Disease 2019 (Covid-19): Men have more Biological Problems, but Women Suffer more Long-Term Serious Psychosocial Consequences and with more Implications for Population. J Women's Health Care 9(3): 487.

36. Turabian JL (2020) The Epidemiological Hypothesis Of "The Trojan Horse": Were Hospitals The Main Vectors Of The Exponencial Beginning Of Coronavirus Disease 2019 (Covid-19) In Spain And Other Countries? Epidemol Int J 4(3): 000146.

37. Wang Y, Tian H, Zhang L, Guo D, Wu W, et al. (2020) Reduction of secondary transmission of SARS-CoV-2 in households by face mask use, disinfection and social distancing: a cohort study in Beijing, China. BMJ Global Health 5: e002794.

38. (2020) Report of the WHO-China Joint Mission on Coronavirus Disease 2019 (COVID-19). World health Organization (WHO).

39. Burke RM, Midgley CM, Dratch A, Fenstersheib M, Haupt T, et al. (2020) Active Monitoring of Persons Exposed to Patients with Confirmed COVID-19-United States, January-February 2020. Morb Mortal Wkly Rep 69(9): 
245-246.

40. Liu Y, Eggo RM, Kucharski AJ (2020) Secondary attack rate and superspreading events for SARS-CoV-2. Lancet 395(10227): e47.

41. Little P, Read RC, Amlot R, Chadborn T, Rice C, et al. (2020) Reducing risks from coronavirus transmission in the home-the role of viral load. BMJ 369: 1728.

42. Zhu J, Zhong Z, Ji P, Li H, Li B, et al. (2020) Clinicopathological characteristics of 8697 patients with COVID-19 in China: a meta-analysis. Family Medicine and Community Health 8(2): e000406.

43. Sun P, Qie S, Liu Z, Ren J, Li K, et al. (2020) Clinical characteristics of hospitalized patients with SARS-CoV-2 infection: A single arm meta-analysis. J Med Virol 92(6): 612-617.

44. Li LQ, Huang T, Wang YQ, Wang ZP, Liang Y, et al. (2020) COVID-19 patients' clinical characteristics, discharge rate, and fatality rate of meta-analysis. J Med Virol 92(6): 577-583.

45. Jarvis C (2020) The Unusual Symptoms of COVID-19. The Scientist.

46. Spinato G, Fabbris C, Polesel J, Cazzador D, Borsetto D, et al. (2020) Alterations in smell or taste in mildly symptomatic outpatients with SARS-CoV-2 infection. JAMA 323(20): 2089-2090.

47. Rimmer A (2020) Anosmia: Five minutes with ENT consultant Claire Hopkins. BMJ 369: 2095.

48. Lechien JR, Chiesa Estomba CM, Hans S, Barillari MR, Jouffe L, et al. (2020) Loss of Smell and Taste in 2013 European Patients With Mild to Moderate COVID-19. Ann Intern Med.

49. Wise J (2020) Covid-19 and thrombosis: what do we know about the risks and treatment?. BMJ 369: 2058.

50. Grens K (2020) Strokes Reported Among Some MiddleAged COVID-19 Patients. The Scientisc.

51. Willyard C (2020) Coronavirus blood-clot mystery intensifies: Research begins to pick apart the mechanisms behind a deadly COVID-19 complication. Nature.

52. Nahum J, Beauchant MT, Daviaud F, Echegut P, Fichet J, et al. (2020) Venous Thrombosis among Critically Ill Patients With Coronavirus Disease 2019 (COVID-19). JAMA 3(5): e2010478.

53. Wang HY, Li XL, Yan ZR, Sun XP, Han J, et al. (2020)

\section{Epidemiology International Journal}

Potential neurological symptoms of COVID-19. Therapeutic Advances in Neurological Disorders 13: 1-2.

54. Lipper GM (2020) 'COVID Toes' and 'Kawasaki' Rash: 5 Cutaneous Signs in COVID-19. Medscape.

55. Lipper GM, Fox LP (2020) Dermatologic Changes With COVID-19: What We Know and Don't Know. Medscape.

56. Devlin H (2020) 'Happy hypoxia': unusual coronavirus effect baffles doctors. Support The Guardian.

57. Cholankeril G, Podboy A, Aivaliotis VI, Kim D, Hsing A, et al. (2020) High Prevalence of Concurrent Gastrointestinal Manifestations in Patients with SARS-CoV-2: Early Experience from California. Gastroenterology.

58. Sultan S, Altayar O, Siddique SM, Davitkov P, Feuerstein JD, et al. (2020) AGA Institute rapid review of the GI and liver manifestations of COVID-19, meta-analysis of international data, and recommendations for the consultative management of patients with COVID-19. Gastroenterology 1(15): 42.

59. Brooks M (2020) COVID-19 Linked to Multiple Cardiovascular Presentations. Medscape.

60. Sommera IE, Bakker PR (2020) What can psychiatrists learn from SARS and MERS outbreaks? Lancet Psychiatry 7: 565-566.

61. Rogers JP, Chesney E, Oliver D, Pollak TA, Fusar Poli P, et al. (2020) Psychiatric and neuropsychiatric presentations associated with severe coronavirus infections: a systematic review and meta-analysis with comparison to the COVID-19 pandemic. The Lancet Psychiatry 7(7): 611-627.

62. Nguyen S, Major K, Cochet C, Bizzozzero T, Barbarossa $\mathrm{L}$, et al. (2020) COVID-19 infection in the elderly in French-speaking Switzerland. An inventory of beliefs, convictions and certainties. Rev Med Suisse 16: 835-838.

63. (2020) Analysis of COVID-19 cases notified to RENAVE until May 10 in Spain: Informe COVID-19 no 33. 29 of May. Red National Epidemiological Surveillance.

64. (2020) Ene-Covid-19 Study: First Round National Study of Seroepidemiology of Sars-Voc-2 Infection in Spain. Instituto Carlos III.

65. Oran DP, Topol EJ (2020) Prevalence of Asymptomatic SARS-CoV-2 Infection. Ann Intern Med.

66. Deng SQ, Peng HJ (2020) Characteristics of and public health responses to the coronavirus disease 2019 outbreak in China. J Clin Med 9(2): 575. 


\section{Epidemiology International Journal}

67. Guo W, Li M, Dong Y, Zhou H, Zhang Z, et al. (2020) Diabetes is a risk factor for the progression and prognosis of COVID-19. Diabetes Metab Res Rev.

68. Cariou B, Hadjadj S, Wargny M, Pichelin M, Al-Salameh A, et al. (2020) Phenotypic characteristics and prognosis of inpatients with COVID-19 and diabetes: the CORONADO Study. Diabetologia, pp: 1-30.

69. Pranata R, Lim MA, Huang I, Raharjo SB, Lukito AA (2020) Hypertension is associated with increased mortality and severity of disease in COVID-19 pneumonia: A systematic review, meta-analysis and meta-regression. J Renin Angiotensin Aldosterone System 21(2): 1-11.

70. Sever P, Johnston SL (2020) The Renin-Angiotensin system and SARS-CoV-2 infection: A role for the ACE2 receptor?. J Renin Angiotensin Aldosterone System 21(2): $1-2$.

71. Jordan RE, Adab P (2020) Who is most likely to be infected with SARS-CoV-2?. Lancet Inf Dis. pp: 1-2.

72. Smith CS, Paauw DS (2000) When you hear hoof beats: four principles for separating zebras from horses. J Am Board Fam Pract 13(6): 424-429.

73. Fernandez TJL, Perez Franco B (2006) The Process by Which Family Doctors Manage Uncertainty: Not Everything Is Zebras or Horses. Primary Care 38(3): 165-167. 